(C) The Authors

Adv. Theor. Math. Phys. 2 (1998) 719 - 731

\title{
A sharp bound for an eigenvalue moment of the one-dimensional Schrödinger operator
}

${ }^{1}$ Dirk Hundertmark ${ }^{\mathrm{a}}$, Elliott H. Lieb ${ }^{\mathrm{a}}$, Lawrence E. Thomas $^{\mathrm{b}}$

\author{
${ }^{a}$ Department of Physics and Mathematics \\ Jadwin Hall \\ Princeton University \\ P.O. Box 708 \\ Princeton \\ New Jersey 08544 \\ ${ }^{b}$ Department of Mathematics \\ University of Virginia \\ Charlottesville \\ Virginia 22903
}

\begin{abstract}
We give a proof of the Lieb-Thirring inequality in the critical case $d=1$, $\gamma=1 / 2$, which yields the best possible constant.
\end{abstract}

e-print archive: http://xxx.lanl.gov/abs/hep-th/9806012

${ }^{1}$ On leave of NWF I - Mathematik, Universität Regensburg, D-93040 Regensburg

(C) The Authors. Reproduction of this article in its entirety, by any means, is permitted for non-commercial purposes. 


\section{Introduction}

There is a family of inequalities [9], [10] that has proved to be useful in various areas of mathematical physics, especially in the proofs of stability of matter. They state that given a Schrödinger operator

$$
-\Delta+V \text { on } \mathrm{L}^{2}\left(\mathbb{R}^{d}\right),
$$

the sum of the moments of the negative eigenvalues $-E_{1}<-E_{2} \leq-E_{3} \leq$ $\ldots \leq 0$ (if any) of this operator is bounded by

$$
\sum E_{i}^{\gamma} \leq L_{\gamma, d} \int\left(V_{-}(x)\right)^{\gamma+d / 2} d x
$$

with $V_{-}(x):=\max (-V(x), 0)$. These inequalities have been generalized in several directions, e.g. manifolds instead of $\mathbb{R}^{d}$. Here we are concerned with the case $d=1$.

The cases originally shown to hold [10] are

$$
d=1, \gamma>\frac{1}{2}, \quad d=2, \gamma>0, \quad \text { and } d \geq 3, \gamma \geq 0 .
$$

When $d=2$ there cannot be any bound for $\gamma=0$ (meaning the number of negative eigenvalues) since at least one negative eigenvalue always exists for arbitrarily small negative perturbations of the free Laplacian in two dimensions [5, page 156-157], [15].

The critical case $d \geq 3$ and $\gamma=0$ was open for a while and proved independently by Cwikel [4], Lieb [7], and Rozenbljum [11]. Still later, different proofs where given by Conlon [3] and $\mathrm{Li}$ and Yau [6]. The sharp constants are still not known, but the best one so far is in [7].

If $d=1$ it is not hard to see that the inequality cannot hold for $\gamma<1 / 2$. To prove this choose a sequence of aproximate $\delta$-functions. They converge to zero in $L^{\gamma+1 / 2}(\mathbb{R})$ but the limit may have a negative eigenvalue; see the discussion of a Dirac potential below. In the critical case $d=1, \gamma=1 / 2$, which concerns us here, it was not known until recently whether $L_{1 / 2,1}$ is finite. This case was settled by Timo Weidl [17] who showed that $L_{1 / 2,1}<$ 1.005. Unfortunately his method of proof cannot be improved to yield the sharp constant as can be seen from the following argument: His method is also applicable for a half-line problem corresponding to a Schrödinger operator on $\mathbb{R}_{+}$with Neumann boundary conditions at the origin; in fact he reduces the full problem (but not the determination of the sharp constant) to this case. Since in this half-line problem the trivial lower bound for the 
sharp constant is given by 1 his method cannot yield a better bound than 1 in the problem concerning us here.

Hence, the sharp constant $L_{1 / 2,1}$ remained undetermined, a tantalizing situation, since there is an obvious conjecture about the value of this constant [10]. In one dimension the potential can be a measure (thanks to the fact that $H^{1}\left(\mathbb{R}^{1}\right)$ functions are continuous) and when $\gamma=1 / 2$ the right hand side of (1) is simply the total mass of this measure. In order to maximize the sum of the square roots of the eigenvalues it is reasonable to suppose that one should concentrate the potential at one point and the extreme case should hence correspond to a $\delta$-function.

It is well-known that $-\partial_{x}^{2}-c \delta$ is a well-defined closed quadratic form on the Sobolev space $H^{1}\left(\mathbb{R}^{1}\right)$ and the Hamiltonian corresponding to this form is used in textbooks as a simple solvable model in quantum mechanics. An exercise shows that the only bound state of this operator for positive $c$ is given by $\psi(x)=\exp (-c|x| / 2)$ with eigenvalue $-c^{2} / 4$.

If it is true that this Dirac potential is the optimal case we conclude that the sharp constant in the Lieb-Thirring inequality for $d=1, \gamma=1 / 2$ is given by $L_{1 / 2,1}=1 / 2$. The proof of this statement is the main result of this paper. A corollary of our result is that for the half-line problem with Neumann boundary conditions considered by Weidl, the sharp constant is 1 .

Before turning to the proof let us note the corresponding - still unproved - conjecture when $1 / 2<\gamma<3 / 2$. The optimal potential should be given by

$$
V(x)=-\frac{1}{\gamma^{2}-1 / 2}\left(\cosh \left(\frac{x}{\gamma^{2}-1 / 2}\right)\right)^{-2}
$$

and the sharp constant is supposed to be [10]

$$
L_{\gamma, 1}=\pi^{-1 / 2} \frac{1}{\gamma-1 / 2} \frac{\Gamma(\gamma+1)}{\Gamma(\gamma+1 / 2)}\left(\frac{\gamma-1 / 2}{\gamma+1 / 2}\right)^{\gamma+1 / 2}=2 L_{\gamma, 1}^{c}\left(\frac{\gamma-1 / 2}{\gamma+1 / 2}\right)^{\gamma-1 / 2} .
$$

Here $L_{\gamma, 1}^{c}:=(2 \pi)^{-1 / 2} \Gamma(\gamma+1) / \Gamma(\gamma+3 / 2)$ is its classical value. Unlike the case $\gamma<3 / 2$ the optimal constant in one dimension and $\gamma \geq 3 / 2$ is known [1], [10] to be $L_{\gamma, 1}=L_{\gamma, 1}^{c}$. Using the fact proved in [1] that $L_{\gamma, 1} / L_{\gamma, 1}^{c}$ is monotone decreasing in $\gamma$ and the sharp value for $L_{1 / 2,1}$ obtained here we conclude that $L_{\gamma, 1} \leq 2 L_{\gamma, 1}^{c}$ for all $\gamma \geq 1 / 2$. As a last remark, let us note that our proof uses no special 1-D technique, except for the explicit form of the Birman-Schwinger kernel (3) in one dimension. 


\section{Proof of the main result for potentials}

The principal result of this paper is

Theorem 1. For a Schrödinger operator $-\partial_{x}^{2}+V$ in one dimension the optimal constant $L_{1 / 2,1}$ is $1 / 2$, i.e.

$$
\sum_{E_{i} \leq 0} \sqrt{E_{i}} \leq \frac{1}{2} \int V_{-}(x) d x
$$

The inequality is strict if the negative part $V_{-}$is a non-zero $\mathrm{L}^{1}$ function.

In this section we prove this theorem in the case the potential is an $\mathrm{L}^{1}$ function. In the last section we extend the bound (2) to potentials that are (finite) measures and prove that the $\delta$-function is the unique maximizer up to translations. By the minmax principle it suffices to investigate the operator $-\partial_{x}^{2}-V_{-}$. We will henceforth assume $V=-U$ with $U$ non-negative and integrable.

To study the bound states energies of a Schrödinger operator it is often useful to investigate another problem. To do so we need some more notation. For $E>0$ let

$$
\mathcal{K}_{E}(x, y):=\sqrt{U(x)} \frac{\exp (-\sqrt{E}|x-y|)}{2 \sqrt{E}} \sqrt{U(y)}, \quad \text { for all } x, y \in \mathbb{R}
$$

be the Birman-Schwinger kernel for the Schrödinger operator $-\partial_{x}^{2}-U$ in $\mathrm{L}^{2}(\mathbb{R}) . \mathcal{K}_{E}$ stands for the integral operator given by this kernel. The BirmanSchwinger principle $[2,13]$ states that $-E_{n}<0$ is the $n^{\text {th }}$ eigenvalue of $-\partial_{x}^{2}-U$ if and only if the $n^{\text {th }}$ eigenvalue of $\mathcal{K}_{E_{n}}$ equals one. The explicit expression (3) suggests that multiplying (3) by $\sqrt{E_{n}}$ will yield a still implicit but perhaps more flexible expression for $\sqrt{E_{n}}$. This is exactly what we are going to do. Let us define, for $\mu \geq 0$,

$$
\mathcal{L}_{\mu}(x, y):=\sqrt{U(x)} \mathrm{e}^{-\mu|x-y|} \sqrt{U(y)}, \quad \text { for all } x, y \in \mathbb{R} .
$$

Moreover, given some arbitrary non-negative locally finite Borel-measure $\kappa$ on $\mathbb{R}$, we can generalize the kernel (4) to

$$
\mathcal{L}^{\kappa}(x, y):=\sqrt{U(x)} \mathrm{e}^{-|\mathcal{J}(x)-\mathcal{J}(y)| \sqrt{U(y)},} \text { for all } x, y \in \mathbb{R},
$$

where the function $\mathcal{J}$ is given by

$$
\mathcal{J}(x):=\int_{0}^{x} \kappa(d z)
$$


Again $\mathcal{L}_{\mu}$ and $\mathcal{L}^{\kappa}$ are the corresponding integral operators. Of course, $\mathcal{L}_{\mu}$ in (4) corresponds to $\kappa(d z)=\mu d z$. Both $\mathcal{K}_{E}$ and $\mathcal{L}^{\kappa}$ are compact integral operators; their Hilbert-Schmidt norms are bounded by $\left(\int U(x) d x\right)^{2} /(2 \sqrt{E})$ and $\left(\int U(x) d x\right)^{2}$, respectively. For a positive compact operator $A$ we denote its ordered eigenvalues by $\lambda_{1}(A) \geq \lambda_{2}(A) \geq \ldots \geq 0$. With the help of the Fourier transform $\left(\exp (-\varepsilon|x|) /(2 \varepsilon)=\int \mathrm{e}^{i p x} /\left(p^{2}+\varepsilon^{2}\right) d p /(2 \pi)\right)$ one sees the following facts:

(i) $\mathcal{L}^{\kappa}$ and $K_{E}$ are positive definite operators, and hence the (ordered) eigenvalues $\lambda_{j}\left(\mathcal{L}^{\kappa}\right)$ obey

(ii) $\lambda_{1}\left(\mathcal{L}^{\kappa}\right)>\lambda_{2}\left(\mathcal{L}^{\kappa}\right) \geq \lambda_{3}\left(\mathcal{L}^{\kappa}\right) \geq \ldots \geq 0$

with a similar statement for $\lambda_{j}\left(\mathcal{K}_{E}\right)$. The strict inequality follows from the positivity of the integral kernel and the Perron-Frobenius theorem. The trace of $\mathcal{L}^{\kappa}$ is given by

(iii) $\operatorname{tr} \mathcal{L}^{\kappa}=\int U(x) d x$,

independent of $\kappa$, and

(iv) $\mathcal{L}^{0}=\mathcal{L}_{0}$ is a rank one operator with eigenvalue $\int U(x) d x$.

The discussion above suggests that the sum of the square roots of the eigenvalues of the one dimensional Schrödinger operator is related to the sum of the eigenvalues of $\mathcal{L}_{\mu}$. Indeed we have the following bound:

Theorem 2 (Domination by $\mathcal{L}_{\mu}$ ). Suppose $U \geq 0$ with $U \in \mathrm{L}^{1}(\mathbb{R})$ and let $-E_{1}<-E_{2} \leq-E_{3} \leq \ldots \leq 0$ be the negative eigenvalues counting multiplicity of the Schrödinger operator $-\partial_{x}^{2}-U$ given by the minmax principle. Furthermore, we denote by $\lambda_{j}\left(\mathcal{L}_{\mu}\right)$ the eigenvalues of $\mathcal{L}_{\mu}$ in (4). Then, for all $n \in \mathbb{N}$ and $0 \leq E \leq E_{n}$

$$
2 \sum_{i \leq n} \sqrt{E_{i}} \leq \sum_{i \leq n} \lambda_{i}\left(\mathcal{L}_{\sqrt{E}}\right)+\lambda_{1}\left(\mathcal{L}_{\sqrt{E_{1}}}\right)-\lambda_{1}\left(\mathcal{L}_{\sqrt{E_{2}}}\right) .
$$

In (7) we set $E_{j+1}=0$ in case the Schrödinger operator happens to have only $j$ negative eigenvalues.

Proof. As already mentioned, the Birman-Schwinger principle gives a one-toone correspondence between negative eigenvalues of a Schrödinger operator and the eigenvalues of $\mathcal{K}_{E}: \lambda_{i}\left(\mathcal{K}_{E_{i}}\right)=1$. Multiplying this equality by $2 \sqrt{E_{i}}$ yields $2 \sqrt{E_{i}}=2 \sqrt{E_{i}} \lambda_{i}\left(\mathcal{K}_{E_{i}}\right)=\lambda_{i}\left(\mathcal{L}_{\sqrt{E_{i}}}\right)$ for all $i$ such that $E_{i}>0$. Note that $\lambda_{i}\left(\mathcal{L}_{0}\right)=0$ if $i \geq 2$ since $\mathcal{L}_{0}$ is a rank one operator. Therefore we have

$$
2 \sum_{i \leq n} \sqrt{E_{i}}=\sum_{i \leq n} \lambda_{i}\left(\mathcal{L}_{\sqrt{E_{i}}}\right)
$$


for arbitrary $n \in \mathbb{N}$. If the eigenvalues of $\mathcal{L}_{\mu}$ were monotonically decreasing as $\mu \geq 0$ increases this would immediately imply

$$
2 \sum_{i \leq n} \sqrt{E_{i}} \leq \sum_{i \leq n} \lambda_{i}\left(\mathcal{L}_{\sqrt{E_{n}}}\right) \leq \sum_{i \leq n} \lambda_{i}\left(\mathcal{L}_{\sqrt{E}}\right) \quad \text { for } 0 \leq E \leq E_{n} .
$$

However, such a monotonicity cannot hold since the trace of $\mathcal{L}_{\mu}$ is independent of $\mu \geq 0$. Nevertheless, the partial sums $\sum_{i \leq n} \lambda_{i}\left(\mathcal{L}_{\sqrt{E}}\right)$ of its eigenvalues are monotone in $E$ even for the slightly more general operator $\mathcal{L}^{\kappa}$ given by (5). Lemma 4 below is the key lemma in our analysis. Assuming the monotonicity given in Lemma 4, the proof of the theorem follows immediately from (8): For $n=1$ we have

$$
\begin{aligned}
2 \sqrt{E_{1}} & =\lambda_{1}\left(\mathcal{L}_{\sqrt{E_{1}}}\right)=\lambda_{1}\left(\mathcal{L}_{\sqrt{E_{2}}}\right)+\lambda_{1}\left(\mathcal{L}_{\sqrt{E_{1}}}\right)-\lambda_{1}\left(\mathcal{L}_{\sqrt{E_{2}}}\right) \\
& \leq \lambda_{1}\left(\mathcal{L}_{\sqrt{E}}\right)+\lambda_{1}\left(\mathcal{L}_{\sqrt{E_{1}}}\right)-\lambda_{1}\left(\mathcal{L}_{\sqrt{E_{2}}}\right) \quad \text { for all } 0 \leq E \leq E_{1}
\end{aligned}
$$

where we take $E_{2}=0$ if the potential has only one negative eigenvalue. If there are two or more negative eigenvalues it follows by induction that

$$
\begin{array}{r}
2 \sum_{i \leq n} \sqrt{E_{i}}+2 \sqrt{E_{n+1}} \\
\leq \sum_{i \leq n} \lambda_{i}\left(\mathcal{L}_{\sqrt{E_{n+1}}}\right)+\lambda_{n+1}\left(\mathcal{L}_{\sqrt{E_{n+1}}}\right)+\lambda_{1}\left(\mathcal{L}_{\sqrt{E_{1}}}\right)-\lambda_{1}\left(\mathcal{L}_{\sqrt{E_{2}}}\right) \\
\leq \sum_{i \leq n+1} \lambda_{i}\left(\mathcal{L}_{\sqrt{E}}\right)+\lambda_{1}\left(\mathcal{L}_{\sqrt{E_{1}}}\right)-\lambda_{1}\left(\mathcal{L}_{\sqrt{E_{2}}}\right)
\end{array}
$$

for all $0 \leq E \leq E_{n+1}$ and $n \in \mathbb{N}$.

Before proving the Lemma, we note a simple consequence of this theorem which proves our main bound (2).

Corollary 3 (Sharp constant). Under the hypotheses of Theorem 2 and for $U \neq 0$

$$
2 \sum_{i \in \mathbb{N}} \sqrt{E_{i}}<\int U(x) d x
$$

Proof. From the theorem we get

$$
\begin{aligned}
2 \sum_{i \in \mathbb{N}} \sqrt{E_{i}} & \leq \lambda_{1}\left(\mathcal{L}_{0}\right)+\lambda_{1}\left(\mathcal{L}_{\sqrt{E_{1}}}\right)-\lambda_{1}\left(\mathcal{L}_{\sqrt{E_{2}}}\right) \\
& =\int U(x) d x+\lambda_{1}\left(\mathcal{L}_{\sqrt{E_{1}}}\right)-\lambda_{1}\left(\mathcal{L}_{\sqrt{E_{2}}}\right)
\end{aligned}
$$


since $\mathcal{L}_{0}$ is a rank one operator with eigenvalue $\int U(x) d x$. To conclude the strict inequality also note that $\lambda_{1}\left(\mathcal{L}_{\sqrt{E}}\right)$ is strictly monotone decreasing in $E \geq 0$ by Lemma 4 . The Perron-Frobenius theorem [12, Theorem XIII.44] implies $E_{1}$ is simple and hence $\lambda_{1}\left(\mathcal{L}_{\sqrt{E_{1}}}\right)-\lambda_{1}\left(\mathcal{L}_{\sqrt{E_{2}}}\right)<0$.

Lemma 4 (Monotonicity). For all $n \in \mathbb{N}$ the $n^{\text {th }}$ partial sum of the eigenvalues of the operator $\mathcal{L}^{\kappa}$ defined in (5) is monotonically decreasing in the sense that

$$
\sum_{i \leq n} \lambda_{i}\left(\mathcal{L}^{\kappa^{\prime}}\right) \leq \sum_{i \leq n} \lambda_{i}\left(\mathcal{L}^{\kappa}\right)
$$

if $\kappa^{\prime}([s, t]) \geq \kappa([s, t])$ for all $s \leq t \in \mathbb{R}$. Moreover the largest eigenvalue $\lambda_{1}\left(\mathcal{L}^{\kappa}\right)$ is strictly monotone decreasing in $\kappa$.

Proof. To clarify the line of reasoning we consider first a toy-model given by an $(m+1) \times(m+1)$ matrix where the two variables $x$ and $y$ in (5) take on $m+1$ values $x_{0} \leq \ldots \leq x_{m}$. With $a_{i}=\exp \left(-\mid J\left(x_{i}\right)-J\left(x_{0} \mid\right)\right) \leq 1$ (where $J$ is defined in (6) and with $U=1$ on $\left\{x_{0}, \ldots, x_{m}\right\}$ for simplicity) the operator given in (5) has the matrix

$$
L\left(\left\{a_{i}\right\}\right):=\left(\begin{array}{cccccc}
1 & a_{1} & a_{1} a_{2} & a_{1} a_{2} a_{3} & \ldots & a_{1} \ldots a_{m} \\
a_{1} & 1 & a_{2} & a_{2} a_{3} & \ldots & a_{2} \ldots a_{m} \\
\vdots & & & & & \vdots \\
a_{1} \ldots a_{m-1} & a_{2} \ldots a_{m-1} & \ldots & & 1 & a_{m} \\
a_{1} \ldots a_{m} & a_{2} \ldots a_{m} & \ldots & & a_{m} & 1
\end{array}\right) .
$$

Let $\lambda_{1}\left(\left\{a_{i}\right\}\right) \geq \lambda_{2}\left(\left\{a_{i}\right\}\right) \geq \ldots \geq \lambda_{m+1}\left(\left\{a_{i}\right\}\right)$ be the ordered eigenvalues of $L\left(\left\{a_{i}\right\}\right)$. We investigate the sum of the largest $n$ eigenvalues in the cube given by $0 \leq a_{k} \leq 1$ for all $k \in\{1, \ldots, m+1\}$ and want to show that it is a (separately) monotone increasing function of each $a_{k}$ in the interval $0 \leq a_{k} \leq 1$. Fix $k \in\{1, \ldots, m+1\}$ and $\left\{a_{i}\right\}_{i \neq k}$. For simplicity we write $L\left(a_{k}\right)$ for $L\left(\left\{a_{i}\right\}_{i \neq k}, a_{k}\right)$. The matrix $L$ has the form

$$
L\left(a_{k}\right):=L\left(\left\{a_{i}\right\}_{i \neq k}, a_{k}\right)=\left(\begin{array}{cc}
A & a_{k} W \\
a_{k} W^{\dagger} & B
\end{array}\right)=: L(0)+a_{k} T
$$

with

$$
L(0):=L\left(\left\{a_{i}\right\}_{i \neq k}, 0\right)=\left(\begin{array}{cc}
A & 0 \\
0 & B
\end{array}\right) \quad \text { on } \mathbb{C}^{k} \oplus \mathbb{C}^{m+1-k}=\mathbb{C}^{m+1}
$$

and the perturbation

$$
T=\left(\begin{array}{cc}
\mathbf{0} & W \\
W^{\dagger} & \mathbf{0}
\end{array}\right), \quad W: \mathbb{C}^{n+1-k} \rightarrow \mathbb{C}^{k}
$$


where $A, B$, and $W$ are $k \times k,(m+1-k) \times(m+1-k)$, and $k \times(m+1-k)$ matrices respectively, depending only on $\left\{a_{i}\right\}_{i \neq k}$. This shows that the dependence of $L$ on $a_{k}$ (for fixed $\left\{a_{i}\right\}_{i \neq k}$ ) is affine-linear. Now the claimed monotonicity of the sum of the largest $n$ eigenvalues in $0 \leq a_{k} \leq 1$ is easily seen by the usual quantum mechanics textbook arguments of perturbation theory, cf. [16, chapter 3.5]: The sum is given by

$$
\begin{aligned}
\sum_{i \leq n} \lambda_{i}\left(L\left(a_{k}\right)\right) & =\sup _{0 \leq d \leq 1, \operatorname{tr} d=n} \operatorname{tr}\left(d L\left(a_{k}\right)\right) \\
& =\sup _{0 \leq d \leq 1, \operatorname{tr} d=n}\left\{\operatorname{tr}\left(d L(0)+a_{k} \operatorname{tr}(d T)\right\}\right.
\end{aligned}
$$

where $d: \mathbb{C}^{m+1} \rightarrow \mathbb{C}^{m+1}$ is a density matrix. Consequently, being a supremum of affine-linear functions, it is convex. To conclude monotonicity in $a_{k}$ it is enough to show that the derivative of the sum with respect to $a_{k}$ at $a_{k}=0$ is non-negative. If the eigenvalues of $L(0)$ are non-degenerate this follows immediately from the Feynmann-Hellman theorem of perturbation theory: Since $L(0)$ leaves the decomposition $\mathbb{C}^{n+1}=\mathbb{C}^{k} \oplus \mathbb{C}^{m+1-k}$ invariant its eigenvectors $\Phi_{i}$ live either in the subspace $\mathbb{C}^{k}$ or $\mathbb{C}^{m+1-k}$, so $\left\langle\Phi_{i}, T \Phi_{i}\right\rangle=0$. Thus by the Feynman-Hellman formula each eigenvalue has derivative 0 at $a_{k}=0$, and for this reason each partial sum has zero derivative at $a_{k}=0$.

In the degenerate case a single eigenvalue might have a negative derivative at $a_{k}=0$ but the partial sum of the largest $n$ eigenvalues always has a non-negative derivative. Indeed, if the eigenvalues are degenerate we first have to diagonalize the perturbation $T$ in the corresponding eigenspace $h$ of $L(0)$. This eigenspace, however, can be decomposed into $h=h_{1} \oplus h_{2}$, with $h_{1} \subset \mathbb{C}^{k}, h_{2} \subset \mathbb{C}^{m+1-k}, h_{1}$ or $h_{2}$ possibly empty. With $P_{i}$ being the orthogonal projection onto $h_{i}, i=1,2$, the perturbation $T$ restricted to the subspace $h$ is again of the form $\left.T\right|_{h}=P_{h} T P_{h}=\widetilde{W}+\widetilde{W}^{\dagger}$, i.e., $\left.T\right|_{h}=\left(\begin{array}{cc}0 & \widetilde{W} \\ \widetilde{W}^{\dagger} & 0\end{array}\right)$ with $\widetilde{W}:=P_{h_{1}} W P_{h_{2}}: h_{2} \rightarrow h_{1}$. This gives $\operatorname{tr}_{h} T=\left.\operatorname{tr} T\right|_{h}=0$. The FeynmanHellman formula tells us that the eigenvalues of the restricted perturbation $\left.T\right|_{h}$ are the derivatives of the eigenvalue branches emerging from this degeneracy subspace at $a_{k}=0$. Since even the perturbation restricted to the eigenspace $h$ has trace zero, we conclude that the derivative of the sum at $a_{k}=0$ is at most greater or equal to zero.

For the strict monotonicity of the largest eigenvalue $\lambda_{1}\left(L\left(\left\{a_{i}\right\}\right)\right)$ in the cube $0<a_{i} \leq 1, i \in\{1, \ldots, m+1\}$ note that by the Frobenius-Perron theorem the corresponding eigenvector $\Phi\left(\left\{a_{i}\right\}\right)$ has only positive entries. Consequently for $0<a_{i}<a_{i}^{\prime} \leq 1$, all $i \in\{1, \ldots, m+1\}$, the minmax 
principle implies

$$
\begin{aligned}
\lambda_{1}\left(L\left(\left\{a_{i}\right\}\right)\right) & =\left\langle\Phi\left(\left\{a_{i}\right\}\right), L\left(\left\{a_{i}\right\}\right) \Phi\left(\left\{a_{i}\right\}\right)\right\rangle \\
& <\left\langle\Phi\left(\left\{a_{i}\right\}\right), L\left(\left\{a_{i}^{\prime}\right\}\right) \Phi\left(\left\{a_{i}\right\}\right)\right\rangle \\
& \leq\left\langle\Phi\left(\left\{a_{i}^{\prime}\right\}\right), L\left(\left\{a_{i}^{\prime}\right\}\right) \Phi\left(\left\{a_{i}^{\prime}\right\}\right)\right\rangle=\lambda_{1}\left(\left\{a_{i}^{\prime}\right\}\right)
\end{aligned}
$$

Remark: The above reasoning for the toy model remains valid if $L$ is replaced by $M L M$ where $M$ is a multiplication operator, i.e. a diagonal matrix, so that the partial sums of the eigenvalues for $M L M$ are also monotone. To apply this reasoning to our operator $\mathcal{L}_{\mu}$ it is enough to show the monotonicity (10) for finite discrete measures $\kappa=\sum c_{j} \delta_{x_{j}}$ and $\kappa^{\prime}=\sum c_{j}^{\prime} \delta_{x_{j}}$ with $c_{j}^{\prime} \geq c_{j}$. Indeed, approximate $\kappa$ and $\kappa^{\prime}-\kappa$ by finite sums $\kappa_{m}$ and $\Delta_{m}$ of $\delta$-functions. This is possible since they are weakly dense in the set of locally finite Borel-measures. It is easy to see that the corresponding operators $\mathcal{L}^{\kappa_{m}}$ and $\mathcal{L}^{\kappa_{m}+\Delta_{m}}$ converge in Hilbert-Schmidt norm to $\mathcal{L}^{\kappa}$ and $\mathcal{L}^{\kappa^{\prime}}$. Monotonicity of the partial sums of eigenvalues of $\mathcal{L}^{\kappa}$ for arbitrary $\kappa$ then follows by approximation and, without loss of generality, we may assume

$$
\kappa=\sum_{j=1}^{m} c_{j} \delta_{x_{j}}, \quad \kappa^{\prime}=\sum_{j=1}^{m} c_{j}^{\prime} \delta_{x_{j}} \quad \text { for some } m \in \mathbb{N}
$$

with $c_{j}^{\prime} \geq c_{j} \geq 0, j \in\{1, \ldots, m\}$, and $-\infty<x_{1}<\ldots<x_{m}<\infty$. For $x \leq y$ we infer

$$
|J(x)-J(y)|=\int_{x}^{y} \kappa(d z)=\sum_{x \leq x_{j} \leq y} c_{j}
$$

and

$$
\begin{aligned}
\mathcal{L}^{\kappa_{m}}(x, y) & =\sqrt{U(x)} \exp \left(-\sum_{x \leq x_{j} \leq y} c_{j}\right) \sqrt{U(y)} \\
& =\prod_{x \leq x_{j} \leq y} \mathrm{e}^{-c_{j}} \sqrt{U(x)} \sqrt{U(y)} \\
& =\prod_{x \leq x_{j} \leq y} a_{j} \sqrt{U(x)} \sqrt{U(y)}, \quad a_{j}:=\mathrm{e}^{-c_{j}}, j=1, \ldots, m \\
& =: \mathcal{L}\left(\left\{a_{j}\right\}\right)(x, y) .
\end{aligned}
$$

As in the matrix case the dependence of $\mathcal{L}\left(\left\{a_{i}\right\}\right)$ on a single $a_{k}$ (for fixed $\left.\left\{a_{j}\right\}_{j \neq k}\right)$ is affine-linear and decomposition of the Hilbert space is now given by $\mathrm{L}^{2}(\mathbb{R})=\mathrm{L}^{2}\left(-\infty, x_{k}\right) \oplus \mathrm{L}^{2}\left(x_{k}, \infty\right)$. Hence we are in precisely the same situation as for our $M L M$ toy-model, and we infer that the partial sums of 
the largest eigenvalues are monotone in $\kappa$ for $\mathcal{L}^{\kappa_{m}}$. By the above limiting argument therefor for $\mathcal{L}^{\kappa}$ and in particular for $\mathcal{L}_{\mu}$.

Strict monotonicity of the largest eigenvalue $\lambda_{1}\left(\mathcal{L}^{\kappa}\right)$ in $\kappa$, i.e. $\lambda_{1}\left(\mathcal{L}^{\kappa^{\prime}}\right)<$ $\lambda_{1}\left(\mathcal{L}^{\kappa}\right)$ if $\kappa^{\prime}>\kappa$, follows from the Perron-Frobenius theorem, the minmax principle, and the strict monotonicity of the kernel (5) in $\kappa$. One can, however, avoid the minmax principle in this conclusion. The Perron-Frobenius theorem states that the eigenvectors $\Phi_{1}^{\kappa}$ and $\Phi_{1}^{\kappa^{\prime}}$ corresponding to $\lambda_{1}\left(\mathcal{L}^{\kappa}\right)$ and $\lambda_{1}\left(\mathcal{L}^{\kappa^{\prime}}\right)$ are non-negative and strictly positive on the support of the potential $U$. By definition

$$
\lambda_{1}\left(\mathcal{L}^{\kappa}\right) \Phi_{1}^{\kappa}=\mathcal{L}^{\kappa} \Phi_{1}^{\kappa}
$$

and the same for $\kappa^{\prime}$. From this we get

$$
\lambda_{1}\left(\mathcal{L}^{\kappa^{\prime}}\right)\left\langle\Phi_{1}^{\kappa}, \Phi_{1}^{\kappa^{\prime}}\right\rangle-\lambda_{1}\left(\mathcal{L}^{\kappa}\right)\left\langle\Phi_{1}^{\kappa^{\prime}}, \Phi_{1}^{\kappa}\right\rangle=\left\langle\Phi_{1}^{\kappa}, \mathcal{L}^{\kappa^{\prime}} \Phi_{1}^{\kappa^{\prime}}\right\rangle-\left\langle\Phi_{1}^{\kappa^{\prime}}, \mathcal{L}^{\kappa} \Phi_{1}^{\kappa}\right\rangle .
$$

since $\left\langle\Phi_{1}^{\kappa}, \Phi_{1}^{\kappa^{\prime}}\right\rangle>0$ and the scalar products in (12) are real, hence symmetric, we get by interchanging the integration variables

$$
\begin{aligned}
\lambda_{1}\left(\mathcal{L}^{\kappa^{\prime}}\right)-\lambda_{1}\left(\mathcal{L}^{\kappa}\right) & =\frac{1}{\left\langle\Phi_{1}^{\kappa}, \Phi_{1}^{\kappa^{\prime}}\right\rangle} \iint \Phi_{1}^{\kappa}(x) \Phi_{1}^{\kappa^{\prime}}(y)\left(\mathcal{L}^{\kappa^{\prime}}(x, y)-\mathcal{L}^{\kappa}(x, y)\right) d x d y \\
& <0
\end{aligned}
$$

by the strict monotonicity of the kernel $\mathcal{L}^{\kappa}(x, y)$ in $\kappa$ and the strict positivity of $\Phi_{1}^{\kappa}, \Phi_{1}^{\kappa^{\prime}}$ on the support of $U$. This concludes the proof of the monotonicity lemma.

\section{Extension to 'potentials' that are measures}

In this section we extend theorem 1 to measure perturbations of $-\partial_{x}^{2}$. As mentioned in the introduction the Sobolev inequality in one dimension, $\mathrm{cf}$. [8][Theorem 8.5], ensures that a finite measure $\tau$ on $\mathbb{R}$ yields a quadratic form $\tau[\phi]:=\int|\phi(x)|^{2} \tau(d x)$ that is infinitesimally form bounded with respect to the Laplacian in one dimension. The quadratic form

$$
\begin{aligned}
\langle\psi, H \phi\rangle & =\left\langle\psi,-\partial_{x}^{2} \phi\right\rangle+\langle\psi, \tau \phi\rangle \\
& :=\left\langle\partial_{x} \psi, \partial_{x} \phi\right\rangle+\int_{\mathbb{R}} \overline{\psi(x)} \phi(x) \tau(d x)
\end{aligned}
$$

is thus closed on the Sobolev space $H^{1}(\mathbb{R})$ and defines a unique self-adjoint operator $H=-\partial_{x}^{2}+\tau$ on $\mathrm{L}^{2}(\mathbb{R})$. By the minmax principle for forms it is again enough to consider the case $\tau=-\nu$ for some positive bounded measure $\nu$ on $\mathbb{R}$. We will hence consider $H=-\partial_{x}^{2}-\nu$. Our result is 
Theorem 5. Suppose $\nu$ is a non-negative measure with $\nu(\mathbb{R})<\infty$ and let $-E_{1}<-E_{2} \leq-E_{3} \leq \ldots \leq 0$ be the negative eigenvalues counting multiplicity of the Schrödinger operator $-\partial_{x}^{2}-\nu$ (if any) given by the corresponding quadratic form. Then

$$
\sum_{i=1}^{\infty} \sqrt{E_{i}} \leq \frac{1}{2} \nu(\mathbb{R})
$$

with equality if and only if the measure $\nu$ is a single Dirac measure.

Proof. One obstacle in the proof of this theorem is to construct an analog of the Birman-Schwinger kernel (3) for measures. It is given by

$$
\widetilde{K}_{E}[\nu](x, y):=\int \frac{1}{\sqrt{p^{2}+E}}(x, \zeta) \frac{1}{\sqrt{p^{2}+E}}(\zeta, y) \nu(d \zeta)
$$

where we set $p^{2}:=-\partial_{x}^{2}$ for convenience. A given measure $\nu$ can be approximated by smooth functions by convoluting it with an approximate $\delta$-function $\nu \rightarrow \nu_{\varepsilon}=\delta_{\varepsilon} * \nu$. Of course $\nu_{\varepsilon} \rightarrow \nu$ weakly and the operators $\widetilde{K}_{E}\left[\nu_{\varepsilon}\right]$ converge to $\widetilde{K}_{E}[\nu]$ for large $E$ in Hilbert-Schmidt norm, hence in the usual operator norm, too. By Tiktopoulos' formula [14] this shows the norm convergence of the resolvents $\left(p^{2}-\nu_{\varepsilon}+E\right)^{-1}$ to $\left(p^{2}-\nu+E\right)^{-1}$ and thus any finite collection of eigenvalues of $p^{2}-\nu_{\varepsilon}$ converges to those of $p^{2}-\nu$. So, applying the results of the last section, we have for any partial sum, i.e. any $n \in \mathbb{N}$

$$
\begin{aligned}
2 \sum_{i \leq n} \sqrt{E_{i}} & \leq \lim _{\varepsilon \rightarrow 0} \int \nu_{\varepsilon}(x) d x+\lim _{\varepsilon \rightarrow 0}\left(\lambda_{1}\left(\mathcal{L}_{\sqrt{E_{1}}}\left[\nu_{\varepsilon}\right]\right)-\lambda_{1}\left(\mathcal{L}_{\sqrt{E_{2}}}\left[\nu_{\varepsilon}\right]\right)\right) \\
& =\int \nu(d x)+\lim _{\varepsilon \rightarrow 0}\left(\lambda_{1}\left(\mathcal{L}_{\sqrt{E_{1}}}\left[\nu_{\varepsilon}\right]\right)-\lambda_{1}\left(\mathcal{L}_{\sqrt{E_{2}}}\left[\nu_{\varepsilon}\right]\right)\right)
\end{aligned}
$$

where for $\mu \geq 0$ the operator $\mathcal{L}_{\mu}\left[\nu_{\varepsilon}\right]$ is defined by the right hand side of (4) with $U(x)$ replaced by $\nu_{\varepsilon}(x)$. For any positive bounded measure $\nu$ let $\widetilde{\mathcal{L}}_{\mu}[\nu]=2 \mu\left(p^{2}+\mu^{2}\right)^{-1 / 2} \nu\left(p^{2}+\mu^{2}\right)^{-1 / 2}$ be defined by its kernel

$$
\widetilde{\mathcal{L}}_{\mu}[\nu](x, y):=2 \mu \int \frac{1}{\sqrt{p^{2}+\mu^{2}}}(x, \zeta) \frac{1}{\sqrt{p^{2}+\mu^{2}}}(\zeta, y) \nu(d \zeta) .
$$

Since the spectrum of an operator of the form $A A^{\dagger}$ is the same as that of $A^{\dagger} A$ except at zero we conclude for $\mu \geq 0$

$$
\lambda_{1}\left(\mathcal{L}_{\mu}\left[\nu_{\varepsilon}\right]\right)=\lambda_{1}\left(\widetilde{\mathcal{L}}_{\mu}\left[\nu_{\varepsilon}\right]\right) \underset{\varepsilon \rightarrow 0}{\longrightarrow} \lambda_{1}\left(\widetilde{\mathcal{L}}_{\mu}[\nu]\right)
$$

since $\lambda_{1}\left(\mathcal{L}_{\mu}\left[\nu_{\varepsilon}\right]\right)>0$ and the operators $\widetilde{\mathcal{L}}_{\mu}\left[\nu_{\varepsilon}\right]$ converge to $\widetilde{\mathcal{L}}_{\mu}[\nu]$ in HilbertSchmidt norm as $\varepsilon \rightarrow 0$. Thus the equivalent of (9) in the measure case is 
given by

$$
2 \sum_{i \in \mathbb{N}} \sqrt{E_{i}} \leq \nu(\mathbb{R})+\lambda_{1}\left(\widetilde{\mathcal{L}}_{\sqrt{E_{1}}}[\nu]\right)-\lambda_{1}\left(\widetilde{\mathcal{L}}_{\sqrt{E_{2}}}[\nu]\right)
$$

By the Perron-Frobenius theorem for quadratic forms we know that the lowest negative eigenvalue $-E_{1}$ of $p^{2}-\nu$ is simple, ie. $E_{1}>E_{2}$. So (14) will follow from (16) once we prove that $0 \leq \mu \mapsto \lambda_{1}\left(\widetilde{\mathcal{L}}_{\mu}[\nu]\right)$ is (strictly) monotone decreasing. The operator $\widetilde{\mathcal{L}}_{\mu}[\nu]$ is given by a strictly positive integral kernel and hence the eigenvector $\phi_{\mu}$ corresponding to the largest eigenvalue is strictly positive. Rewriting $\widetilde{\mathcal{L}}_{\mu}[\nu] \phi_{\mu}=\lambda_{1}\left(\widetilde{\mathcal{L}}_{\mu}[\nu]\right) \phi_{\mu}$ with $\psi_{\mu}=$ $\left(p^{2}+\mu^{2}\right)^{1 / 2} \phi_{\mu}>0$ we get $2 \mu\left(p^{2}+\mu^{2}\right)^{-1} \nu \psi_{\mu}=\lambda_{1}\left(\widetilde{\mathcal{L}}_{\mu}[\nu]\right) \psi_{\mu}$. Consequently for $0 \leq \mu_{1}, \mu_{2}$

$$
\lambda_{1}\left(\widetilde{\mathcal{L}}_{\mu_{1}}[\nu]\right)\left\langle\psi_{\mu_{2}}, \nu \psi_{\mu_{1}}\right\rangle=2 \mu_{1}\left\langle\psi_{\mu_{2}}, \nu \frac{1}{p^{2}+\mu_{1}^{2}} \nu \psi_{\mu_{1}}\right\rangle
$$

and similarly for $\lambda_{1}\left(\widetilde{\mathcal{L}}_{\mu_{2}}[\nu]\right)$ with $\mu_{1}$ and $\mu_{2}$ interchanged. As in the end of the proof of Lemma 4 we can substract these equations and interchange the integration variables to arrive at

$$
\begin{aligned}
\lambda_{1} & \left(\widetilde{\mathcal{L}}_{\mu_{1}}[\nu]\right)-\lambda_{1}\left(\widetilde{\mathcal{L}}_{\mu_{2}}[\nu]\right) \\
& =\frac{1}{\left\langle\psi_{\mu_{1}}, \nu \psi_{\mu_{2}}\right\rangle} \iint \nu(d x) \nu(d y) \psi_{\mu_{1}}(x) \psi_{\mu_{2}}(y)\left[\mathrm{e}^{-\mu_{1}|x-y|}-\mathrm{e}^{-\mu_{2}|x-y|}\right] \\
& <0 \text { for } 0 \leq \mu_{2}<\mu_{1}
\end{aligned}
$$

if $\nu$ is not concentrated at one point.

Acknowledgment: D.H. and L.T. would like to thank the physics department of Princeton university for its warm hospitality and we thank Wolfgang Spitzer for discussions. - The authors also thank the following organizations for their support: Deutsche Forschungsgemeinschaft, grant Hu 773/1-1 (DH), and the U.S. National Science Foundation, grant PHY9513072 A02 (EHL), and grant DMS 9801329 (LET).

\section{References}

[1] M. Aizenmann and E. H. Lieb: On semi-classical bounds for eigenvalues of Schrödinger operators. Phys. Lett. 66A (1978), 427-429.

[2] M. S. Birman: The spectrum of singular boundary problems. Mat. Sb. 55 No.2 (1961), 125-174, translated in Amer. Math. Soc. Trans. (2), 53 (1966), 23-80. 
[3] J. G. Conlon: A new proof of the Cwikel-Lieb-Rosenbljum bound. Rocky Mountain J. Math., 15, no.1 (1985), 117-122.

[4] M. Cwikel: Weak type estimates for singular values and the number of bound states of Schrödinger operators. Trans. AMS, 224 (1977), 93-100.

[5] L. D. Landau and E. M. Lifshitz: Quantum Mechanics. Non-relativistic theory. Volume 3 of Course of Theoretical Physics, Pergamon Press (1958)

[6] P. Li and S.-T. Yau: On the Schrödinger equation and the eigenvalue problem. Comm. Math. Phys., 88 (1983), 309-318.

[7] E. H. Lieb: The number of bound states of one body Schrödinger operators and the Weyl problem. Bull. Amer. Math. Soc., 82 (1976), 751-753. See also Proc. A.M.S. Symp. Pure Math. 36 (1980), 241-252.

[8] E. H. Lieb and M. Loss: Analysis. Graduate Studies in Mathematics 14, American Mathematical Society 1997.

[9] E. H. Lieb and W. Thirring: Bound for the kinetic energy of fermions which proves the stability of matter. Phys. Rev. Lett., 35 (1975), 687-689. Errata 35 (1975), 1116.

[10] E. H. Lieb and W. Thirring: Inequalities for the moments of the eigenvalues of the Schrödinger Hamiltonian and their relation to Sobolev inequalities. Studies in Math. Phys., Essays in Honor of Valentine Bargmann, Princeton (1976),

[11] G. V. Rozenbljum: Distribution of the discrete spectrum of singular differential operators. Dokl. AN SSSR, 202, N 5 1012-1015 (1972), Izv. VUZov, Matematika, N.1(1976), 75-86.

[12] M. Reed and B. Simon: Methods of modern mathematical physics IV: Analysis of operators. Academic Press, New York 1978.

[13] J. Schwinger: On the bound states of a given potential. Proc. Nat. Acad. Sci. U.S.A. 47, (1961), 122-129.

[14] B. Simon: Quantum mechanics for Hamiltonians defined as quadratic forms. Princeton Series in Physics, Princeton University press, New Jersey, 1971.

[15] B. Simon: The bound state of weakly coupled Schrdinger operators in one and two dimensions. Ann. Physics 97, no. 2, (1976), 279-288.

[16] W. Thirring: A course in mathematical physics. Vol. 3. Quantum mechanics of atoms and molecules. Translated from the German by Evans M. Harrell. Lecture Notes in Physics, 141. Springer-Verlag, New York-Vienna, 1981.

[17] T. Weidl: On the Lieb-Thirring constants $L_{\gamma, 1}$ for $\gamma \geq 1 / 2$. Comm. Math. Phys., 178, no. 1, (1996), 135-146. 